\title{
Extremely low geomagnetic activity during the recent deep solar cycle minimum
}

\author{
E. Echer ${ }^{1}$, B. T. Tsurutani ${ }^{2}$ and W. D. Gonzalez ${ }^{1}$ \\ ${ }^{1}$ National Institute for Space Research (INPE) \\ S. J. Campos, SP, Brazil \\ email: ezequiel.echer@gmail.com, gonzalez@dge.inpe.br \\ ${ }^{2}$ Jet Propulsion Laboratory(JPL), California Institute of Technology (CALTECH) \\ Pasadena, CA, USA \\ email: bruce.tsurutani@jpl.nasa.gov
}

\begin{abstract}
The recent solar minimum (2008-2009) was extreme in several aspects: the sunspot number, $R_{z}$, interplanetary magnetic field (IMF) magnitude $B_{o}$ and solar wind speed $V_{s w}$ were the lowest during the space era. Furthermore, the variance of the IMF southward $B_{z}$ component was low. As a consequence of these exceedingly low solar wind parameters, there was a minimum in the energy transfer from solar wind to the magnetosphere, and the geomagnetic activity $a p$ index reached extremely low levels. The minimum in geomagnetic activity was delayed in relation to sunspot cycle minimum. We compare the solar wind and geomagnetic activity observed in this recent minimum with previous solar cycle values during the space era (1964-2010). Moreover, the geomagnetic activity conditions during the current minimum are compared with long term variability during the period of available geomagnetic observations. The extremely low geomagnetic activity observed in this solar minimum was previously recorded only at the end of XIX century and at the beginning of the XX century, and this might be related to the Gleissberg (80-100 years) solar cycle.
\end{abstract}

Keywords. Sun: activity, Sun: magnetic fields, solar-terrestrial relations, solar wind

\section{Introduction}

The recent solar cycle was the longest with the deepest minimum in sunspot number $\left(R_{z}\right)$ values in the space era (Gibson et al. 2009; Hathaway 2010; Russell, Luhmann \& Jian 2010; de Toma 2011; Echer et al. 2011; Tsurutani, Echer \& Gonzalez 2011). The deep minimum is also reflected in several solar observations, such as solar irradiance (Fröhlich 2009) and radio flux at $10.7 \mathrm{~cm}$ (Hathaway 2010), solar polar magnetic field (Wang, Robbrecht \& Sheeley 2009), and in solar wind parameters at 1 AU (Luhmann et al. 2009; Russell, Luhmann \& Jian 2010; Tsurutani, Echer \& Gonzalez 2011), and in the outer heliosphere (McComas et al. 2008; Smith \& Balogh 2008). The polar coronal holes were smaller in this minimum relative to the previous minimum (Kirk et al. 2009; de Toma 2011). Furthermore, extreme flux enhancements were observed in cosmic rays (Heber et al. 2009; Tsurutani, Echer \& Gonzalez 2011). As a consequence, there have been many geophysical effects, both in the magnetosphere and ionosphere/atmosphere (Gibson et al. 2009; Minamoto \& Taguchi 2009; Kataoka \& Miyoshi 2010; Ram, Lin \& Su 2010; Russell, Luhmann \& Jian 2010; Echer et al. 2011; Emery et al. 2011; Verkhoglyadova et al. 2011). 
Transference of solar wind energy to the magnetosphere depends on the interplanetary magnetic field (IMF) magnitude $B_{o}$, IMF direction and solar wind speed $V_{s w}$ (Gonzalez et al. 1994; Echer et al. 2005). We examine these parameters at $1 \mathrm{AU}$ in addition to the solar wind-magnetosphere energy coupling $\epsilon$ parameter (Perreault \& Akasofu 1978) and the ap geomagnetic activity index (Bartel 1950; Rostoker 1972). Annual averages of geomagnetic indices for periods previous to the space era are also analysed. The study of these parameters will indicate the cause of the low geomagnetic activity during solar minimum.

\section{Methodology}

In this work, we investigate the long term variation of solar wind and geomagnetic parameters. We used 27-day Bartel rotation averages of solar wind parameters from NASA/GSFC solar wind data base (omniweb.gsfc.nasa.gov/). Also, 27-day averages of the sunspot number (Eddy 1976; Echer et al. 2005) and the geomagnetic activity ap index (Rostoker 1972) have been used to assess solar and geomagnetic activity. In order to estimate the solar-wind magnetosphere energy coupling, the $\epsilon$ parameter (Perreault \& Akasofu 1978) is computed. For this calculation, we used high resolution 1-hour solar wind data and then averaged over 27-day Bartel rotations. The $\epsilon$ parameter is calculated as:

$$
\epsilon=10^{7} l_{\mathrm{o}}^{2} V_{\mathrm{sw}} B_{\mathrm{o}}^{2} \sin ^{4}(\theta / 2)[1]
$$

The interplanetary parameters used in Eq. (1) are the IMF magnitude $B_{o}$, the IMF clock angle $\theta$ in the plane perpendicular to the Sun-Earth line, and the solar wind speed $V_{s w}$. The factor $l_{o}$ is an empirically determined scale factor with the physical dimension of length, $7 \mathrm{R}_{E}$, representing the magnetopause radius. The parameter $\epsilon$ is given in $[\mathrm{W}]$.

For the long term evaluation of geomagnetic activity, the annual averages of the $a p$, $A E$, and Dst geomagnetic indices (Bartel 1950; Rostoker 1972), the $a a$ index and the sunspot number $R_{z}$ have been used. The $a p$ and $a a$ index were obtained from the National Geophysical Data Center (www.ngdc.noaa.gov/) and the $R_{z}$ from the Solar Influences Data Center (sidc.oma.be/). The $A E$ and Dst indices were obtained from the World Data Center for Geomagnetism (wdc.kugi.kyoto-u.ac.jp/). The mean magnetic field of the Sun (Scherrer et al. 1977) data was obtained from the Stanford University Wilcox Solar Observatory (wso.stanford.edu).

\section{Results}

\subsection{Space era}

Fig. 1 shows, from top to bottom, the sunspot number $\left(R_{z}\right)$, the solar wind speed $V_{s w}$, the 1 AU interplanetary magnetic field (IMF) magnitude $B_{o}$, the $\epsilon$ parameter, and the ap geomagnetic index.

The data extend from 01 January 1964 to 31 December 2010. This interval covers essentially the entire space era when interplanetary parameters are available. Solar minimum epochs as given by the 13-month smoothed sunspot number are shown in the $R_{z}$ panel as vertical dashed lines. The average of the parameters for \pm 6 months around solar minimum is indicated in Table 1.

Fig. 1 shows that the solar cycle that has just ended (in 2008) is the longest in the space era (12.6 years, extending from 1996 to 2008). The length of cycles 20 to 22 were 11.7, 10.3 and 9.7 years, respectively. The solar minimum $R_{z}$ average for the last cycle is 1.7, which is considerably lower than the other minima: 12.2, 12.3 and 8.0 (Hathaway 


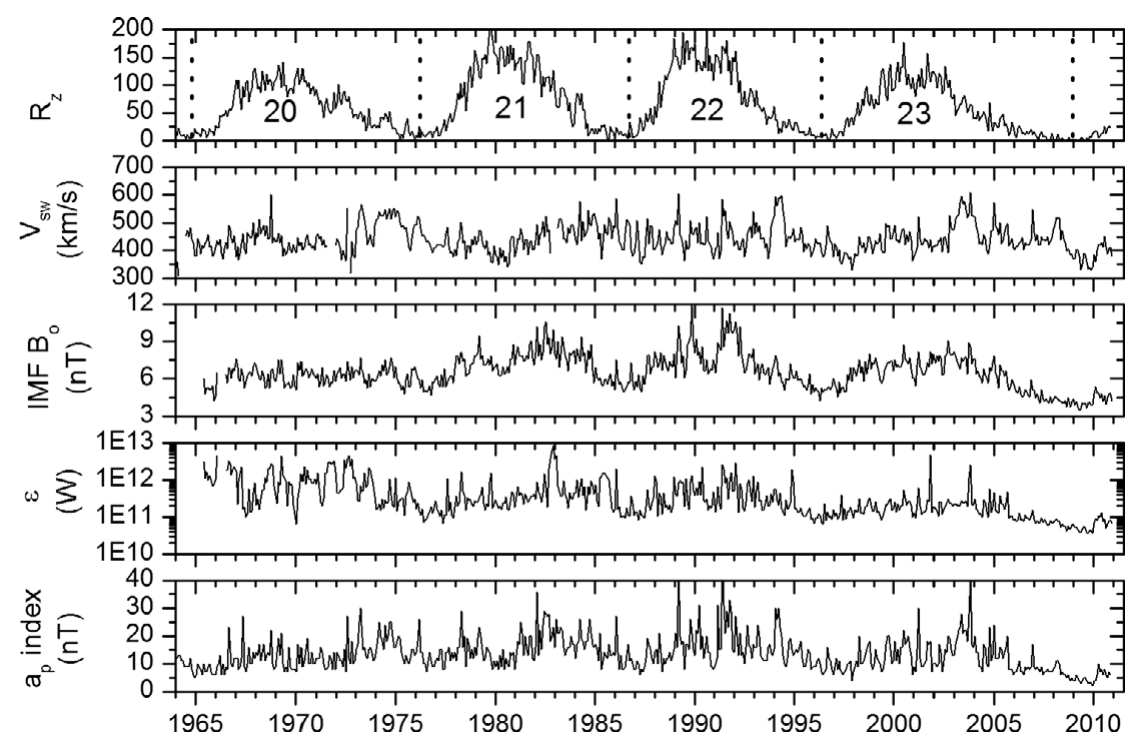

Figure 1. Solar rotation averages of sunspot number, $R_{z}$, solar wind speed $V_{s w}$, interplanetary magnetic field IMF magnitude $B_{o}, \epsilon$ parameter, $a p$ index. Vertical dashed lines in the $R_{z}$ panel indicates the time of solar minima.

Table 1. Average parameters for an interval of \pm 0.5 year around solar cycle minima

\begin{tabular}{ccccccc}
\hline Cycle & Date min (year/month) & $R_{z}$ & $B_{o}(\mathrm{nT})$ & $V_{s w}(\mathrm{~km} / \mathrm{s})$ & $\epsilon\left(10^{11} \mathrm{~W}\right)$ & ap $(\mathrm{nT})$ \\
\hline $19-20$ & $1964 / 10$ & 10.3 & - & 431 & - & 8.5 \\
$20-21$ & $1976 / 03$ & 12.5 & 5.8 & 459 & 1.61 & 13.5 \\
$21-22$ & $1986 / 09$ & 13.1 & 5.7 & 444 & 1.64 & 10.7 \\
$22-23$ & $1996 / 05$ & 8.7 & 5.1 & 419 & 1.09 & 9.5 \\
$23-24$ & $2008 / 12$ & 2.0 & 4.0 & 395 & 0.54 & 4.9 \\
\hline
\end{tabular}

2010). It can be noted that sunspot number, IMF magnitude, solar wind speed and ap index have the lowest averages during the recent past minimum. The average $R_{z}$ value for the period of 1 year around solar minimum is 2.0, lower than the previous four solar cycle minimum values of 8.7 to 13.1 .

Comparison of the IMF magnitude at $1 \mathrm{AU}$ to the $R_{z}$ ( Fig. 1 ) shows that the IMF is more-or-less in phase with the sunspot number, with maximum averages located close in time to the solar maxima. It can be noted that in the current cycle minimum, the IMF average is the lowest on record, about $4 \mathrm{nT}$. In the previous solar minima, the average $\mathrm{IMF}$ magnitude was always $\geqslant 5 \mathrm{nT}$.

The solar wind speed (Fig. 1 third panel) reached maximum values in the solar cycle declining phases in years 1973, 1984, 1994 and 2003. One possible explanation is that the high speed streams coming from coronal holes predominate in this phase of solar cycle (Tsurutani et al. 1995). High speed streams will cause a higher radial IMF component due to the high velocities and the stretching of the Parker spiral. The solar wind speed reached minimum values of $\sim 395 \mathrm{~km} / \mathrm{s}$ during this last cycle minimum, slightly lower than previous cycles (always greater than $400 \mathrm{~km} / \mathrm{s}$ ). The solar rotation averaged ap index shows values of $\sim 5 \mathrm{nT}$ (minimum of $2 \mathrm{nT}$ ) during the current solar minimum, contrasting to values of $\sim 8.5-13.5 \mathrm{nT}$ in previous minima.

Since the energy transferred by the solar wind to the magnetosphere depends on $V_{s w}$ and $B_{o}$, from the results of Table 1 for average $V_{s w}$ and IMF $B_{o}$ one expects that the 
average energy was also smaller during the recent solar cycle minimum as compared to the previous minima. Table 1 shows the average for each solar minimum period. The average power was $\sim 1.1$ to $1.64 \times 10^{11} \mathrm{~W}$ in the three previous solar minima, and $5.4 \mathrm{x}$ $10^{10} \mathrm{~W}$ in the most recent minimum. Thus the energy input to the magnetosphere during this last solar minimum is only $\sim 30 \%$ to $50 \%$ of the value for previous solar cycle minima during space era.

The solar and interplanetary causes in the geomagnetic activity during the last two solar minima were studied (Tsurutani, Echer \& Gonzalez 2011). Minima in geomagnetic activity (MGA) at Earth occurring at the ends of solar cycles (SC) SC23 and SC22 have been identified. Figure 2 shows these MGA intervals. The two MGAs were present in 2009 and 1997, delayed from the sunspot number minima in 2008 and 1996 by 0.5 to 1 years. This is in agreement with geomagnetic activity occurring after sunspot minimum, as seen for example in the $a a$ index (Gao 1986; Kane 2002; Hathaway 2010). For instance, it has been found that, for most of the solar cycles, the minimum in the $a a$ index occurs more than three months later than the sunspot number minimum (Kane 2002).

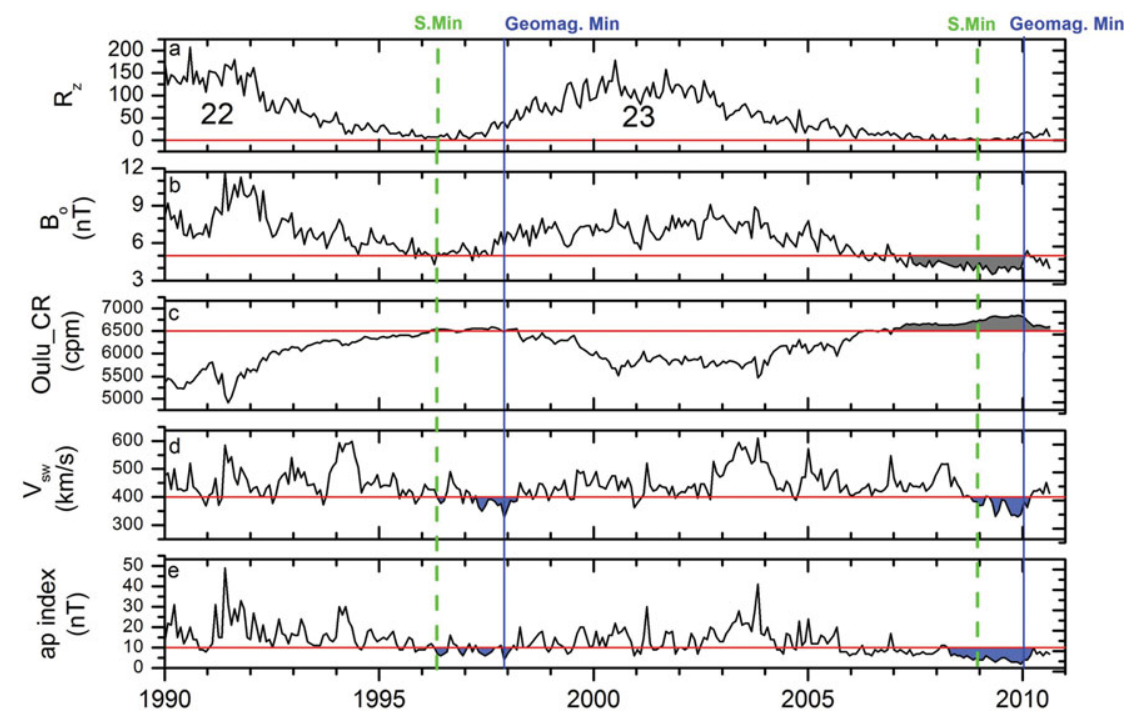

Figure 2. Solar, interplanetary, cosmic ray and geomagnetic activity data for $\mathrm{SC} 22$ and 23 . A dashed vertical green line indicates the official sunspot minima. A vertical solid line shows the geomagnetic activity minima on Earth. Horizontal (arbitrary) red lines are shown for $R_{z}=0$, $B_{o}=5 \mathrm{nT}, \mathrm{CR}=6500 \mathrm{cpm}, V_{s w}=400 \mathrm{~km} \mathrm{~s} 1$ and $a p=10 \mathrm{nT}$. The shaded regions in the $a p$ panel are used to define the minimum geomagnetic activity (MGA) intervals, MGA22 and MGA23. (After Tsurutani, Echer \& Gonzalez 2011).

A detailed comparison between the last two MGA was performed (Tsurutani, Echer \& Gonzalez 2011). It was found that the solar wind speed was about the same for the more recent and the previous cycle (390 vs. $395 \mathrm{~km} / \mathrm{s}$ ), but the IMF $B_{o}$ was much lower in the recent minimum (4.2 versus $5.5 \mathrm{nT}$ ). The geomagnetic activity was $a p=5 \mathrm{nT}$ versus $a p=$ $8.7 \mathrm{nT}$. The cause of the lower IMF $B_{o}$ was the Sun's magnetic field; using Wilcox Observatory/Stanford University observations of the Sun's mean magnetic field, it was found that the solar magnetic field was much weaker in the recent minimum (Tsurutani, Echer \& Gonzalez 2011) . Furthermore, the polar magnetic fields of the Sun are weaker as well (Wang, Robbrecht \& Sheeley 2009; Russell, Luhmann \& Jian 2010; de Toma 2011).

Fig. 3 shows daily averages of sunspot number $R_{z}$, the solar mean magnetic field and the IMF $B_{o}$ for 1975-2010. It can be seen that the solar magnetic field is much weaker 
in this current minimum than in the previous two solar minima. The IMF $B_{o}$ at $1 \mathrm{AU}$ is also a minimum as a consequence. The Sun's field is lower in the last cycle relative to the two previous ones.

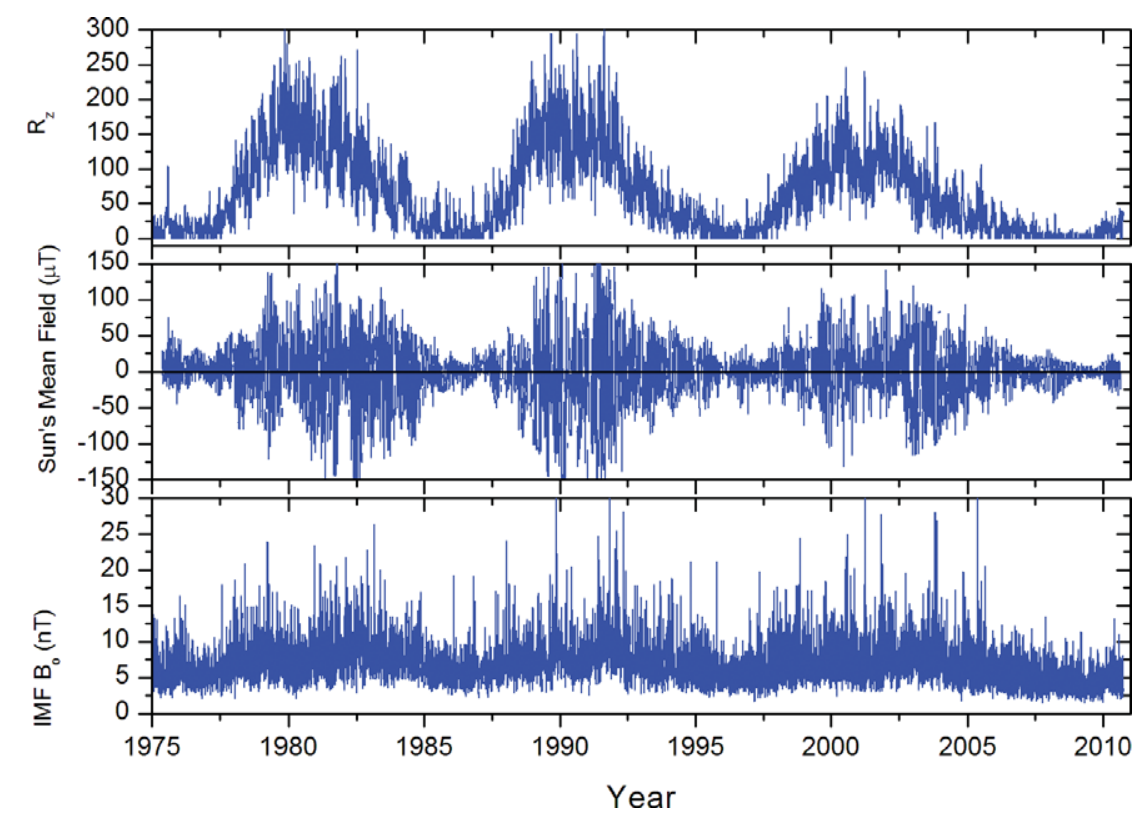

Figure 3. Daily averages of sunspot number, $R_{z}$, Stanford's Sun's mean field and the IMF $B_{o}$ for 1975-2010.

The solar wind speed was very low in the MGA23, with no stream with peak speed higher than $600 \mathrm{~km} / \mathrm{s}$ at Earth's orbit. As it is well known, high speed solar wind streams emanate from coronal holes (Zirker 1977; Tsurutani et al. 1995; Richardson 2006). When the coronal hole is located at low solar latitudes, streams from its center can reach the Earth. When the coronal hole is located at middle latitudes, only the solar wind emanating from the edges of the coronal hole can reach Earth. The velocities at the edges are lower due to superradial expansion of the solar wind. In this recent minimum, there is a sharp contrast between 2008 and 2009 (de Toma 2011). At the end of 2008, the large low latitude coronal holes were closing down and smaller, mid-latitude coronal holes were appearing. In 2009, the disappearance of the low-latitude coronal holes shifted the sources of the high speed solar wind to higher latitudes, mostly to the edges of the solar coronal holes (de Toma 2011). Furthermore, the variances of the IMF $B_{z}$ were computed (Tsurutani, Echer \& Gonzalez 2011). They found that the nested and normalized nested variances (e.g., Tsurutani et al. 1982) are lower in the recent minimum than in the previous one and are also lower than intervals of high speed streams in year 2003 (Tsurutani, Echer \& Gonzalez 2011).

The location of the coronal holes relative to the ecliptic plane led to low solar wind speeds and low interplanetary magnetic field $B_{z}$ variances (and normalized variances) at Earth. Since the coupling of energy transfer between the solar wind and the magnetosphere depends on the IMF $B_{o}$, the IMF $B_{z}$ fluctuations and the velocity of the solar wind, there was reduced solar wind-magnetospheric energy coupling during 2009, leading to the lowest geomagnetic ap index in its recording history. 


\subsection{Historical data}

It is important to assess how unprecedented is the very low geomagnetic activity observed in the recent solar minimum. We assess the geomagnetic activity using data from the historical period with instrumental record, since the XIX century.

Fig. 4 shows the whole interval of ap (since 1932), and $D s t$ and $A E$ indices (since 1957). Also shown for comparison is the $R_{z}$. It can be seen that the current minimum in geomagnetic ap index is the lowest in the history of ap (see also Minamoto \& Taguchi 2009). This indicates that solar wind-magnetosphere energy transfer during the current minimum was very low.

The $A E$ index also shows lowest values during this period. As it is known that longterm averages of $A E$ have high correlation with the ap index (Echer et al. 2004), this would be expected. The $D s t$ index shows very low (in magnitude) values, the lowest in the space era, and comparable to another period of low values during the 1960s (Minamoto \& Taguchi 2009).

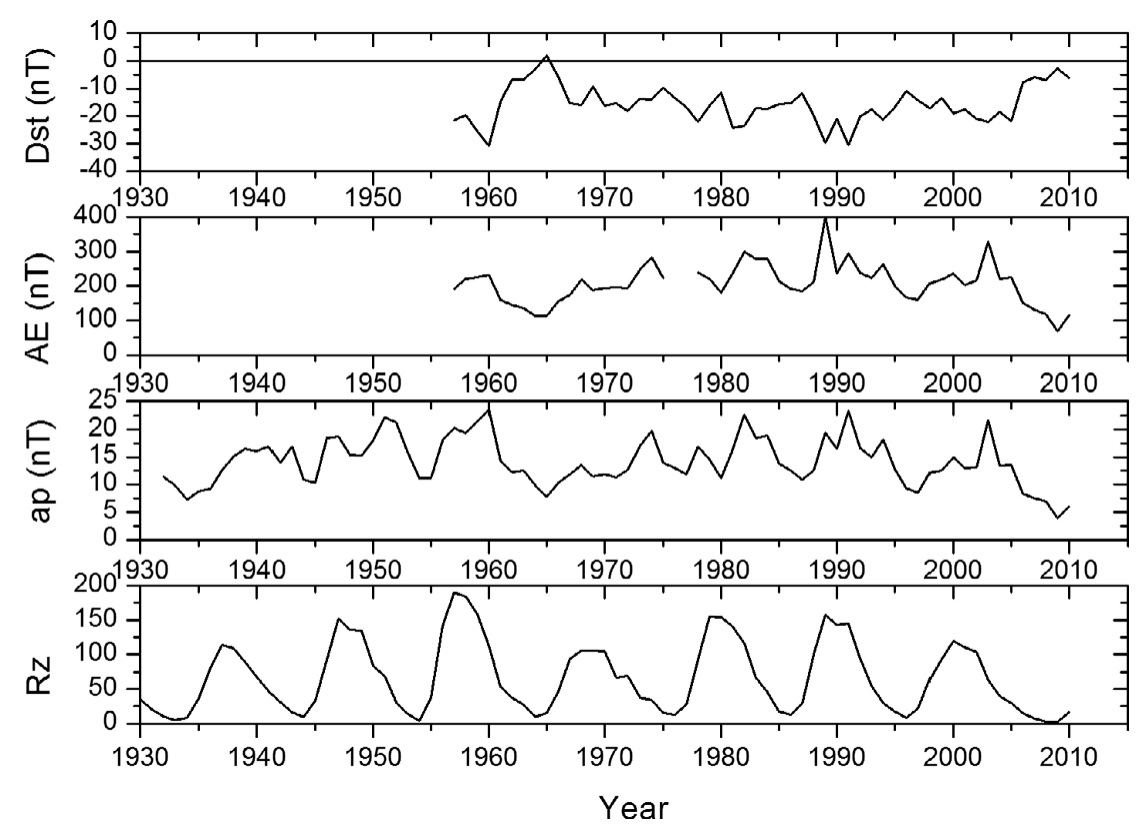

Figure 4. Yearly averages of geomagnetic indices: Dst, $A E$, and $a p$, and the sunspot number $R_{z}$.

Fig. 5 shows the annual averages of $R_{z}$ and the $a a$ index for 1868-2010. It can be seen that in the recent minimum the $a a$ index was the lowest in the entire XX century and the first decade of XXI century, and it is comparable only to the low geomagnetic acitvity in the end of XIX century, when solar activity was lower. The recent minimum in $R_{z}$ is also very low for the XX-XXI century, being compared only to low solar activity seen in the beginning of XX century (de Toma 2011).

The long term correlation between the geomagnetic $a a$ index and $R_{z}$ was found to be decreasing since the beginning of aa index recording in 1868 (Echer et al. 2004). Geomagnetic activity has a dual peak distribution, with one peak near solar maximum and other in the declining phase (Gonzalez, Gonzalez \& Tsurutani 1990). The cause for the decrease of correlation between $R_{z}$ and $a a$ has been attributed to fact that the second a a peak is becoming stronger relative to the first one, which could be due to the fact 


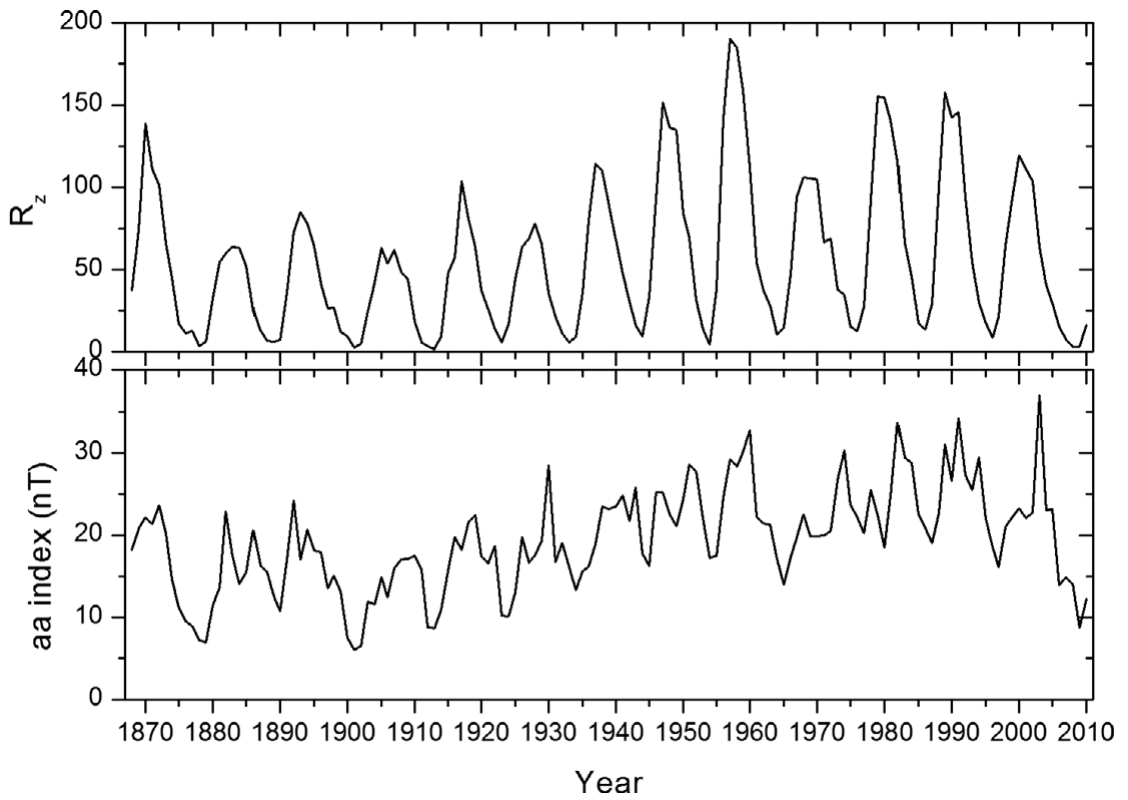

Figure 5. Yearly averages of sunspot number $R_{z}$ (top) and $a a$ index (bottom) for the interval 1868-2010.

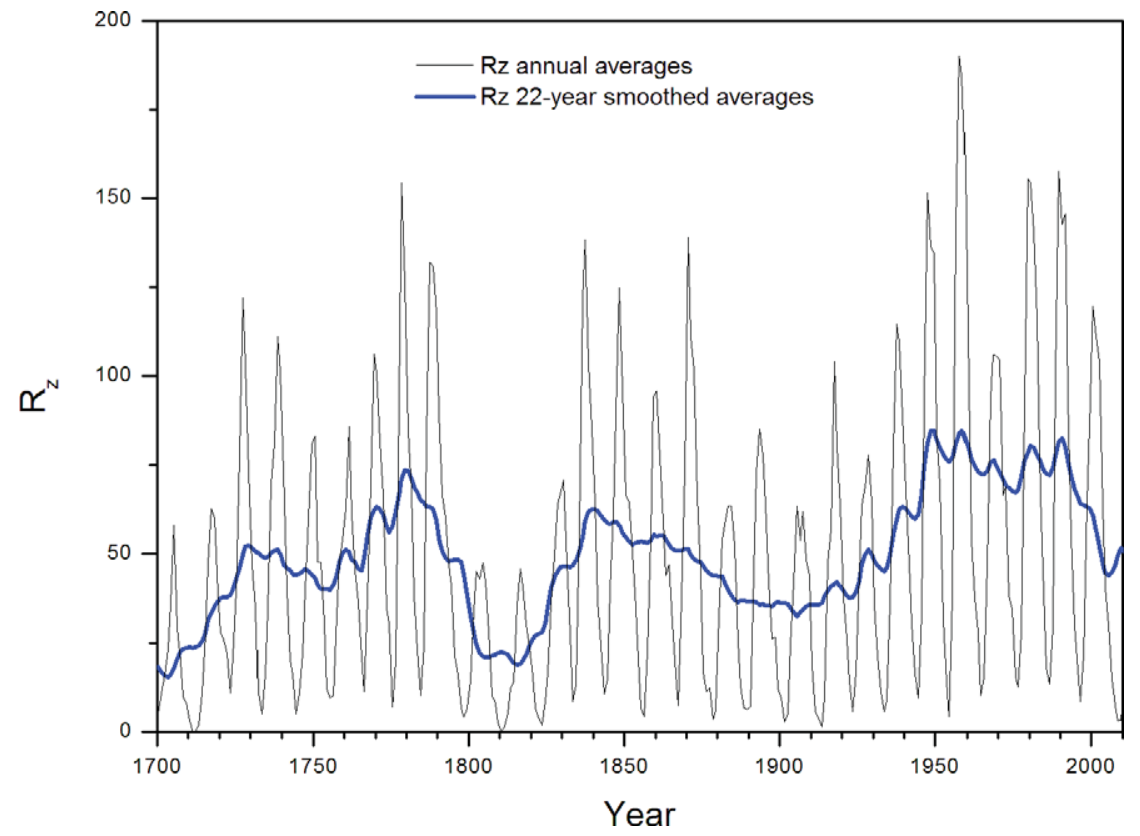

Figure 6. Yearly averages of sunspot number $R_{z}$ (black lines), and a 22-year smoothed curve of $R_{z}$ (blue lines) for the interval 1700-2010.

that solar open magnetic field structures (the aa 2nd peak) seemed to have increase their strength relative to closed magnetic activity structures ( $a a$ 1rst peak, $R_{z}$ related) due to long term variability of the Sun's magnetic field (Stamper et al. 1999; Echer et al. 2004).

Fig. 6 shows the sunspot number and its 22-year smoothed curve for 1700-2010. This long term variation could be related to the Gleissberg cycle of solar activity (Hathaway 2010; Gonzalez et al. 2011). There are 8 to 10 solar cycles between consecutive minima 
of the modulation. The last two Gleissberg minima occurred approximately around 1900 and 2010 (maxima at 1840 and 1960), as can be seen in Figure 6. Thus the current minimum might be a result of the combination of a minimum in the Schwabe 11-year cycle and the Gleissberg minima, which could contributed to make this minimum deeper. The lowest values in $a a$ index also occurred in the two periods of Gleissberg minima, around 1900 and 2010.

\section{Conclusions}

This past solar cycle minimum has been extreme in several aspects. The sunspot number $\left(R_{z}\right)$ was the lowest in recent history. Associated with this characteristic, the duration of cycle 23 has been elongated in comparison to cycles 20 through 22 . The solar wind speed $V_{s w}$ was a minimum of $330 \mathrm{~km} / \mathrm{s}$ (average $395 \mathrm{~km} / \mathrm{s}$ ) and the embedded IMF $B_{o}$ was an exceptionally low $\sim 4 \mathrm{nT}$. These low values of IMF $B_{o}$ and $V_{s w}$ caused a decrease in the energy transfer from solar wind to the magnetosphere, as estimated by the $\epsilon$ parameter, which was 30-50\% of previous solar cycle minima, causing the lowest value of space era in geomagnetic activity as quantified by ap index.

The solar wind magnetosphere energy transference depends on the solar wind speed $V_{s w}$, the IMF $B_{o}$ and the IMF $B_{z}$ component. The energy transfer, estimated by computing the $\epsilon$ parameter, was the lowest of the space era, and the cause is the combination of low solar wind speeds, low IMF magnitude and low IMF $B_{z}$ variances (Alfvenic fluctuations). The low IMF $B_{o}$ values at $1 \mathrm{AU}$ are consequence of low solar fields, as observed for example in solar magnetograms. The $V_{s w}$ is low because of the location of the CHs. The $B_{z}$ component is low also because of the location of the CHs.

The recent solar minimum is also different from previous ones, being characterized by small midlatitude coronal holes and polar coronal holes, instead of big polar coronal holes extending equatorwards. We surmise that it is the superradial expansion from the small midlatitude coronal holes that gives the feeble high speed solar wind speeds. The minimum in the 11-year sunspot cycle was also coincident with a minimum in the Gleissberg cycle, which could be the cause of the deep minimum in solar activity. This result implies that in extended solar minima such as the Maunder minimum (1645-1715), exceptionally low solar magnetic fields and feeble solar winds and embedded magnetic fields are to be expected. The energy transference to Earth's magnetosphere and atmosphere is therefore expected to be a minimum during such epochs accounting for the lack of auroral sightings during that era.

\section{Acknowledgements}

EE would like to thank the CNPq (PQ-300211/2008-2) agency for financial support. WDG would like to thank FAPESP agency (2008/06650-9) for financial support. Portions of this work were performed at the Jet Propulsion Laboratory, California Institute of Technology under contract with NASA.

\section{References}

Bartels, J. 1950, J. Geophys. Res., 55, 427

de Toma, G. 2011, Solar Phys., 274, 195

Echer, E., Gonzalez, W. D., Gonzalez, A. L. C., Prestes, A., Vieira, L. E. A., Dal Lago, A., Guarnieri, F. L., \& Schuch, N. J. 2004, J. Atmos. Solar-Terr. Phys., 66, 1019

Echer, E., Gonzalez, W. D., Guarnieri, F. L., Dal Lago, A., \& Vieira, L. E. A. 2005, Adv. Space Sci., 35,855 
Echer, E., Tsurutani, B. T., Gonzalez, W. D., \& Kozyra, J. U. 2011, Sol. Phys., 274, 303

Eddy, J. A. 1976, Science, 192, 1189

Emery, B. A., Richardson, I. G., Evans, D. S., Rich, F. J., \& Wilson, G. R. 2011, Solar Phys., 274,399

Fröhlich, C. 2009, Astron. Astrophys., 501, L27

Gao, M. 1986, in: Y. Kamide \& J. A. Slavin (eds.), Solar-Wind Magnetosphere Coupling, (Astrophysics and Space Science Library, 126), p. 149

Gibson, S. E., Kozyra, J. U., de Toma, G., Emery, B. A., Onsager, T., \& Thompson, B. J. 2009, J. Geophys. Res., 114

Gonzalez, W. D., Gonzalez, A. L. C., \& Tsurutani, B. T. 1990, Planet. Space Sci., 38, 181

Gonzalez, W. D, Joselyn, J. A., Kamide, Y., Kroehl, H. W., Rostoker, G., Tsurutani, B. T., \& Vasyliunas, V. M. 1994, J. Geophys. Res., 99, 5771

Gonzalez, W. D., Echer, E., Tsurutani, B. T., Clua de Gonzalez, A. L., \& Dal Lago, A. 2011 Space Sci. Rev., 158, 69

Hathaway, D. H. 2010, Liv. Rev. Solar Phys., 7, 1

Heber, B., Kopp, A., Gieseler, J. , Muller-Mellin, R., Fichtner, H., Scherer, K., Potgieter, M. S., \& Ferreira, S. E. S. 2009, Astrophys. J., 699, 1956

Kane, R. P. 2002, Ann. Geophys., 20, 1519

Kataoka, R. \& Miyoshi, Y. 2010, Space Weather, 8, 1

Kirk, M. S., Pesnell, W. D., Young, C. A., \& Hess Webber, S. A. 2009, Sol. Phys., 257, 99

Luhmann, J. G., Lee, C. O., Li, Y., Arge, C. N., Galvin, A. B., Simunac, K., Russell, C. T., Howard, R. A., \& Petrie, G. 2009, Solar Phys., 256, 285

McComas, D. J., Ebert, R. W., Elliott, H. A., Goldstein, B. E., Gosling, J. T., Schwadron, N. A., \& Skoug, R. M. 2008, Geophys. Res. Lett., 35, L18103

Minamoto, Y. \& Taguchi, Y. 2009, Earth Planets Space, 61, e25

Perreault, P. \& Akasouf, S.-I. 1978, Geophys. J. Royal Astron. Soc., 54, 547

Ram, S. T., Liu, C. H., \& Su, S.-Y. 2010, J. Geophys. Res., 115, A12340

Richardson, I. G. 2006, in: B. T. Tsurutani, R. McPherron, W. Gonzalez, G. Lu, J. H. A. Sobral \& N. Gopalswamy (eds.), Recurrent Magnetic Storms: Corotating Solar Wind Streams (AGU Geophysical Monograph 167), p. 45

Rostoker, G. 1972, Rev. Geophys. Space Phys., 10, 935

Russell, C. T., Luhmann, J. G., \& Jian, K. J. 2010, Rev. Geophys, 48, RG2004

Scherrer, P. H., Wilcox, J. M., Svalgaard, L., Duvall, T. L., Dittmer, H., \& Gustafson, E. K. 1977, Solar Phys., 54, 353

Smith, E. J. \& Balogh, A. 2008, Geophys. Res. Lett., 35, L22103

Stamper, R., Lockwood, M., Wild, M. N., \& Clark, T. D. G. 1999, J. Geophys. Res., 104, 28325

Tsurutani, B. T., Smith, E. J., Pyle, K. R., \& Simpson, J. A. 1982, J. Geophys. Res., 87, 7389

Tsurutani, B. T., Gonzalez, W. D., Gonzalez, A. L. C., Tang, F., Arballo, J. K., \& Okada, M. 1995, J. Geophys. Res., 100, 21717

Tsurutani, B. T., Echer, E., \& Gonzalez, W. D. 2011, Ann. Geophys., 29, 83

Verkhoglyadova, O. P., Tsurutani, B. T., Mannucci, A. J., Mlynczak, M. G., Hunt, L. A., Komjathy, A., \& Runge, T. 2011, J. Geophys. Res., 116, A09325

Wang, Y.-M., Robbrecht, E., \& Sheeley, N. R. 2009, Astrophys. J., 707, 1372

Zirker, J. B. 1977, Rev. Geophys. Space Phys., 15, 257

\section{Discussion}

JANET LuHMANn: ICME fields at minimum are due to magnetic clouds. This cycle had north-leading ICME fields. How does this affect geoactivity, have you taken this into account? Also, there are lower solar wind densities during this minimum. Could this also affect the overall geoactivity?

EZEQUIEL ECHER: ICME fields affect mainly the occurrence of intense storms, which are absent from 2007-2010 interval. However, for long term geomagnetic activity (as solar rotation averages), high speed streams have a stronger effect. The solar wind density 
can affect geomagnetic activity, but the main parameters (interplanetary magnetic field magnitude and southward direct Bz component, solar wind speed) are already low enough to cause this very low geomagnetic activity observed during the recent solar minimum. 